\title{
Sex differences in psychosis: normal or pathological?
}

\author{
Janneke Spauwen $^{\mathrm{a}}$, Lydia Krabbendam ${ }^{\mathrm{a}}$, Roselind Lieb ${ }^{\mathrm{b}}$, Hans-Ulrich Wittchen ${ }^{\mathrm{b}}$, Jim van \\ $\mathrm{Os}^{\mathrm{a}, \mathrm{c}, *}$
}

a Department of Psychiatry and Neuropsychology, azM/Mondriaan/Riagg/RIBW/Vijverdal Academic Centre, Maastricht University, European Graduate School of Neuroscience, P.O. Box 616 (DRT 10), 6200 MD Maastricht, The Netherlands

b Max Planck Institute of Psychiatry, Clinical Psychology and Epidemiology Unit, Kraepelinstrasse 2, D-80804 Munich, Germany

c Division of Psychological Medicine, Institute of Psychiatry, De Crespigny Park, Denmark Hill, London SE5 8AF, UK

\begin{abstract}
Background: Schizophrenia first appears in adolescence, in boys at an earlier age than girls. The interpretation of this key epidemiological finding crucially depends on whether similar age-related sex differences exist in the expression of associated, subclinical psychosis-like experiences. Methods: Findings are based on a population sample of 2548 adolescents and young adults aged 17-28. Subjects were assessed with the core psychosis sections on delusions and hallucinations of the MunichComposite International Diagnostic Interview. Results: The risk of subclinical psychotic experiences was significantly higher for males in the younger half of the cohort (17-21 years), but similar in the older half (22-28 years). Conclusions: These findings suggest that normal maturational changes in adolescence with differential age of onset in boys and girls cause the expression of psychosis, the extreme of which is schizophrenia.
\end{abstract}

Keywords: Sex differences; Psychosis; Normal population; Adolescents

The dramatic increase in the incidence of schizophrenia after puberty occurs at an earlier age in boys than in girls, causing the prevalence of the disorder to be higher in boys than in girls during adolescence (WHO, 1992; Konnecke et al., 2000). This epidemiological imbalance is thought to reflect sex differences in the timing of maturational events that facilitate the onset of psychotic disorder (Galdos et al., 1993; Kraepelin, 1919).

One way to further elucidate the meaning of this key epidemiological finding is to examine whether a similar age-related sex difference exists in the expression of subtler, nonclinical but much more frequent psychotic experiences such as low-grade delusional ideas or auditory hallucinations that are continuous with the disorder but do not require treatment (Poulton et al., 2000).

Several studies have shown that it is possible to measure, in the general population, the attenuated but structurally similar psychotic experiences that are encountered in clinical samples (van Os et al., 2001; Peters et al., 1999; Verdoux et al., 1998b). These subclinical manifestations of hallucinations and delusions have been shown to display similar associations with risk factors and demographic variables as have been reported for the clinical psychotic disorder (van Os et al., 2001; Verdoux et al., 1998a; Maric et al., 2003). Therefore, if the nonclinical psychotic experiences increase differentially with age in boys and girls in the same fashion as the clinical disorder schizophrenia (Fig. 1B), that is, if boys have a 
significantly earlier onset of subclinical symptoms than girls, a likely explanation would be that a normal, sex-related maturational event after puberty causes expression of psychosis along a continuum of severity. The implication of this would be that expression of lower grade psychotic experiences arises through the same mechanism as the disorder schizophrenia.

However, if the sex differences in age of onset of schizophrenia are not reflected in similar sex differences in the age of onset of nonclinical psychotic experiences (Fig. 1A), it is unlikely that these normal maturational events themselves play a causal role in the expression of psychosis. Instead, they may merely facilitate the causal influence of a rare, early brain abnormality that is more common in males and remained "dormant" until after puberty as postulated in the neurodevelopmental hypothesis of schizophrenia (Murray and Lewis, 1988; Waddington et al., 1999; Weinberger, 1987).

\section{Subjects and methods}

\subsection{Sample}

The Early Developmental Stages of Psychopathology (EDSP) study (Lieb et al., 2000) collected data on the prevalence, incidence, risk factors, comorbidity and course of mental disorders in a random representative population sample of adolescents and young adults (age range 14-24 years) in the Munich area (Germany). The overall design of the study is prospective, consisting of a baseline survey $(n=3021)$, two follow-up surveys and a family supplement. Fourteen- to fifteen-year-olds were sampled at twice the rate of persons 16-21 years of age, and 22-24- year-olds were sampled at half this rate. A complete and detailed description of design, sample, instruments, procedures and statistical methods of the EDSP is given elsewhere (Wittchen et al., 1998).

The baseline sample was drawn in 1994 from the government registries in Munich, Germany, of registrants expected to be 14-24 years of age at the time of the baseline interview in 1995 . Details about the sampling and representativeness of the whole EDSP sample, along with its sociodemographic characteristics, have been previously presented (Lieb et al., 2000; Wittchen et al., 1998). A total of 3021 interviews were completed at baseline (T0; response rate, 71\%). The first follow-up study (T1) was conducted only for respondents aged 14-17 years at baseline, whereas the second follow-up study was conducted for all respondents. The current results are based on the second follow-up. From the 3021 respondents of the baseline study, a total of 2548 interviews were completed at the second follow-up (T2), which occurred at an average of 42 months after baseline (response rate, $84 \%$ ).

\subsection{Instruments}

Respondents were assessed with the computer-assisted version of the Munich-Composite International Diagnostic Interview (M-CIDI) (Wittchen, 1997), an updated version of the World Health Organization's Composite International Diagnostic Interview version 1.2 (WHO, 1990). The CIDI is designed for use by trained interviewers who are not clinicians and have high interrater reliability (Cottler et al., 1991; Wittchen et al., 1991) and high testretest reliability (Wittchen, 1994). Most interviews were carried out in the homes of the respondents. Trained and highly experienced clinical interviewers at the level of psychologist conducted all interviews. Interviewers were allowed to probe if necessary. At baseline, the lifetime version of the M-CIDI was used. At each of the follow-up assessments, we applied the MCIDI interval version, which refers to the period of assessment from the last interview 
until the present. Data on the M-CIDI G-section about psychosis were only present for the T2 assessment, so these data are lifetime ratings.

\subsection{Psychosis ratings}

The ratings from the $15 \mathrm{M}$-CIDI core psychosis sections on delusions (11 items) and hallucinations (4 items) were used (items G3-G5, G7-G14, G17, G18, G20, G21). These concern classic psychotic symptoms involving, for example, persecution, thought interference and auditory hallucinations. All of these items can be rated in two ways: 1, no and 5, yes. The presence of positive psychotic experiences was broadly defined as any rating of 5 on any of the $15 \mathrm{M}$-CIDI core psychosis items.

\subsection{Statistical analysis}

All analyses were carried out using the software package STATA, version 7 (StataCorp, 2001). Logistic regression analysis was performed in order to examine the association between any positive psychotic symptom on the one hand and age and sex on the other. The interaction between age and sex was assessed using the likelihood ratio test. A p-value $<0.05$ was considered statistically significant. In order to investigate whether any associations between sex and psychotic experiences were confounded by differences in substance abuse between boys and girls, the analyses were adjusted for substance abuse and separately for cannabis use as the most frequently used drug.

\section{Results}

The response rate at the second follow-up was $84.3 \%(\mathrm{n}=2548)$. The sample consisted of 1299 males $(50.9 \%)$ and the mean age was 21.7 years (S.D. $=3.4)$. The cumulative incidence of positive psychotic experiences was $17.5 \%$, similar to previous population-based studies (van Os et al., 2001). Thus, 15.7\% reported having had at least one delusional experience and $4.6 \%$ reported having had at least one hallucinatory experience. In those who reported at least one psychotic experience, the mean age was lower in boys $(21.3$ years, S.D. $=3.3)$ than in girls (22.4 years, S.D. $3.5, \mathrm{t}=-2.9, \mathrm{df}=440, \mathrm{P}=0.0042)$.

Logistic regression indicated that there was no main effect of age (in years) or sex on the presence of at least one positive symptom (OR 1.00, 95\% CI $0.97-1.03$ and OR $0.92,95 \%$ CI $0.75-1.13$, respectively). However, a significant interaction between age and sex was present (likelihood ratio test: $\chi^{2}=6.7, p=0.0097$ ). Stratified analysis revealed that in the younger half of the cohort (split around the median age of 21 years), the risk of having a positive psychotic symptom was higher for males than for females $(\mathrm{OR}=0.70,95 \% \mathrm{CI} 0.52-0.95)$, while no sex difference was found in the older cohort (OR $=1.18,95 \%$ CI $0.89-1.58)$. The finding of differential sex ratios by age group remained even after controlling for substance abuse (interaction likelihood ratio test: $\chi 2=7.7, p=0.006$ ) and separately for cannabis use (interaction likelihood ratio test: $\chi 2=7.8, \mathrm{p}=0.005$ ).

\section{Discussion}

Nonclinical experiences of psychosis showed differential sex distributions by age that resembled those reported for the clinical disorder schizophrenia. This suggests that sex differences in psychosis may reflect differential age-related variation of a continuous phenotype, rather than differential expression of a rare causal pathological risk factor. 
This contrasts with some previous hypotheses, in particular, that a rare early brain anomaly interacts with normal maturation to produce schizophrenia (Weinberger, 1987). The findings may be compatible with the hypothesis proposed by Feinberg (Keshavan et al., 1994) that variation in normal maturational processes may be related to the expression of psychosis. According to Feinberg, abnormalities in the elimination of synapses in the prefrontal cortex that takes place during adolescence may result in psychotic symptom formation. Adolescence is a critical period for the development of positive psychotic symptoms both in clinical and nonclinical samples (Verdoux et al., 1998b; Hafner et al., 1993).

Age-related sex differences in these maturational processes, in particular, a significantly greater loss of cerebral grey matter in boys compared to girls (De Bellis et al., 2001), may represent the underlying mechanism of males showing an earlier age of onset of psychosis than females in a nonclinical sample, similar to results found in clinical samples.

Raine (1992), Maric et al. (2003) and Tien (1991) reported that women have more positive "paranormal" and psychosis-like experiences than men. This seems to contradict our findings. However, our study focussed on differential sex ratios by age group in adolescents and young adults. In contrast, the studies carried out by Raine (1992), Maric et al. (2002) and Tien (1991) focussed on main effects of sex on the prevalences of psychotic experiences across the entire adult age range. Therefore, we feel that our findings do not oppose these reports. In fact, each of these studies provides support for the hypothesis that associations with demographic risk factors in schizophrenia are mirrored in similar associations at lower levels of the continuum: In the current study, the sex ratio in the older adult part of our sample increased and rose well above unity, a directionally similar change as the ones reported by Raine (1992), Maric et al. (2002) and Tien (1991).

A limitation of this work concerns the use of self-reported symptoms, although the use of face-to-face interviewing by psychologists with enough skills to ask follow-up questions and probe, if necessary, can be expected to yield a much better result than a self-report questionnaire or even a CIDI interview by trained lay interviewers. In the M-CIDI, the assessment of the symptoms does not include the degree of distress or disability associated with symptoms. Respondents who only have minor distress cannot be distinguished from respondents who have major distress. However, it has been demonstrated that psychotic or psychosis-like symptoms may occur in the population without any accompanying distress (van Os et al., 2001).

Furthermore, previous work has shown that these self-reported experiences are valid as they are both aetiologically (van Os et al., 2001) and longitudinally (Poulton et al., 2000) on a continuum with clinical psychotic disorders.

The use of cumulative incidence by age at interview may arguably represent another limitation. However, the age of the current population is so young that it is reasonable to assume that psychotic experiences were fairly recent in the majority of respondents. Even if the sample would include subjects who had their first psychotic experiences a few years ago, it is unlikely that this would have led to spurious results. In addition, a post hoc analysis in which the sample was restricted to those with psychotic experiences during the last year yielded essentially similar results (interaction likelihood ratio test: $\chi^{2}=3.7, p=0.05$ ).

In conclusion, two implications flow from these observations. First, the findings suggest that nonclinical and clinical psychotic experiences in adolescence are expressed along a 
continuum of severity. Second, brain maturational changes in adolescence with differential timing in males and females may cause expression of psychosis along the entire continuum.

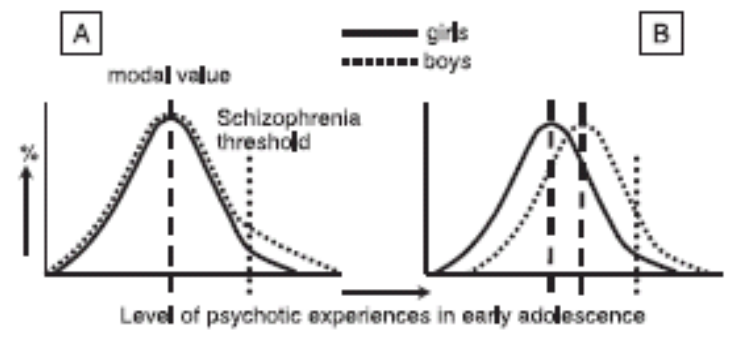

Fig. 1. Models explaining higher prevalence of schizophrenia in boys during adolescence. If the sex difference in the level of psychotic experiences in adolescence (higher level in males) apply to schizophrenia but not to the less severe, nonclinical expressions of psychosis (A), it is unlikely that sex-related post-pubertal maturation events causally influence the expression of psychosis. However, if the entire distribution of adolescent expression of psychosis is shifted (B), it is likely that some nomal maturational event with differential onset in boys and girls causes expression of psychosis.

\section{Acknowledgements}

The EDSP is funded by the German Ministry of Research and Education (BMBF) as part of the Addiction Research Initiative (01EB94056).

\section{References}

- Cottler, L.B., Helzer, J.E., Mager, D., Spitznagel, E.L., Compton, W.M., 1991. Agreement between DSM-III and III-R substance use disorders. Drug Alcohol Depend. 29, $17-25$.

- $\quad$ De Bellis, M.D., Keshavan, M.S., Beers, S.R., Hall, J., Frustaci, K., Masalehdan, A., Noll, J., Boring, A.M., 2001. Sex differences in brain maturation during childhood and adolescence. Cereb. Cortex 11, 552- 557.

- Galdos, P.M., van Os, J.J., Murray, R.M., 1993. Puberty and the onset of psychosis. Schizophr. Res. 10, 7-14.

- Hafner, H., Maurer, K., Loffler, W., Riecher-Rossler, A., 1993. The influence of age and sex on the onset and early course of schizophrenia. Br. J. Psychiatry 162, 80-86.

- Keshavan, M.S., Anderson, S., Pettegrew, J.W., 1994. Is schizophrenia due to excessive synaptic pruning in the prefrontal cortex? The Feinberg hypothesis revisited. J. Psychiatr. Res. 28, 239- 265.

- Konnecke, R., Hafner, H., Maurer, K., Loffler, W., an der Heiden, W., 2000. Main risk factors for schizophrenia: increased familial loading and pre- and perinatal complications antagonize the protective effect of oestrogen in women. Schizophr. Res. 44, 81-93.

- Kraepelin, E., 1919. Dementia Preacox and Paraphrenia (translated by M. Barclay). Churchill Livingstone, Edinburgh.

- $\quad$ Lieb, R., Isensee, B., von Sydow, K., Wittchen, H.U., 2000. The Early Developmental Stages of Psychopathology Study (EDSP): a methodological update. Eur. Addict. Res. 6, 170-182.

- Maric, N., Krabbendam, L., Vollebergh, W., de Graaf, R., van Os, J., 2003. Sex differences in symptoms of psychosis in a nonselected general population sample. Schizophr. Res. (in press).

- Murray, R.M., Lewis, S.W., 1988. Is schizophrenia a neurodevelopmental disorder? Br. Med. J. (Clin. Res. Ed.) 296, 63. 
- Peters, E.R., Joseph, S.A., Garety, P.A., 1999. Measurement of delusional ideation in the normal population: introducing the PDI (Peters et al. Delusions Inventory). Schizophr. Bull. 25, 553-576.

- Poulton, R., Caspi, A., Moffitt, T.E., Cannon, M., Murray, R., Harrington, H., 2000. Children's self-reported psychotic symptoms and adult schizophreniform disorder: a 15-year longitudinal study. Arch. Gen. Psychiatry 57, 1053-1058.

- Raine, A., 1992. Sex differences in schizotypal personality in a nonclinical population. J. Abnorm. Psychol. 101, 361-364.

- StataCorp, 2001. Stata Statistical Software: Release 7.0. Stata Corporation, College Station, TX.

- Tien, A.Y., 1991. Distributions of hallucinations in the population. Soc. Psychiatry Psychiatr. Epidemiol. 26, 287- 292.

- $\quad$ van Os, J., Hanssen, M., Bijl, R.V., Vollebergh, W., 2001. Prevalence of psychotic disorder and community level of psychotic symptoms: an urban- rural comparison. Arch. Gen. Psychiatry 58, 663-668.

- Verdoux, H., Maurice-Tison, S., Gay, B., van Os, J., Salomon, R., Bourgeois, M.L., 1998a. A survey of delusional ideation in primary-care patients. Psychol. Med. 28, 127- 134.

- Verdoux, H., van Os, J., Maurice Tison, S., Gay, B., Salamon, R., Bourgeois, M., 1998b. Is early adulthood a critical developmental stage for psychosis proneness? A survey of delusional ideation in normal subjects. Schizophr. Res. 29, 247-254.

- Waddington, J.L., Lane, A., Larkin, C., O'Callaghan, E., 1999. The neurodevelopmental basis of schizophrenia: clinical clues from cerebro-craniofacial dysmorphogenesis, and the roots of a lifetime trajectory of disease. Biol. Psychiatry 46, 31-39.

- Weinberger, D.R., 1987. Implications of normal brain development for the pathogenesis of schizophrenia. Arch. Gen. Psychiatry 44, 660-669.

- WHO, 1990. Composite International Diagnostic Interview (CIDI), Version 1.0. World Health Organisation, Geneva, Switzerland.

- WHO, 1992. International Classification of Diseases, 10th ed. World Health Organisation, Geneva.

- Wittchen, H.U., 1994. Reliability and validity studies of the WHO-Composite International Diagnostic Interview (CIDI): a critical review. J. Psychiatr. Res. 28, 57-84.

- Wittchen, H.U., 1997. DIA-X-Interviews: Manual für Screening-Verfahren und Interview; Interviewheft Längsschnittuntersuchung (DIA-X-Lifetime); Ergänzungsheft (DIA-XLifetime); Interviewheft Querschnittsuntersuchung (DIA-X-12 Monats-Version); Ergänzungsheft (DIA-X-12 Monats-Version); PC-Programm zur Durchführung der Interviews (Längs- und Querschnittsuntersuchung). Auswertungsprogramm. Frankfurt, Germany: Swets and Zeitlinger.

- Wittchen, H.U., Robins, L.N., Cottler, L.B., Sartorius, N., Burke, J.D., Regier, D., 1991. Cross-cultural feasibility, reliability and sources of variance of the Composite International Diagnostic Interview (CIDI). The Multicentre WHO/ADAMHA Field Trials. Br. J. Psychiatry 159, 645-653, 658.

- Wittchen, H.U., Nelson, C.B., Lachner, G., 1998. Prevalence of mental disorders and psychosocial impairments in adolescents and young adults. Psychol. Med. 28, 109-126. 